\title{
Effect of Liraglutide on Times in Glycaemic Ranges as Assessed by CGM for Type 2 Diabetes Patients Treated With Multiple Daily Insulin Injections
}

\author{
Sheyda Sofizadeh (1) - Henrik Imberg · Arndís F. Ólafsdóttir • \\ Magnus Ekelund · Sofia Dahlqvist · Irl Hirsch • Karin Filipsson • \\ Bo Ahrén · Stefan Sjöberg · Jaako Tuomilehto · Marcus Lind
}

Received: June 18, 2019 / Published online: September 28, 2019

(C) The Author(s) 2019

\begin{abstract}
Introduction: The effects of the GLP-1 analogue liraglutide on time in hypoglycaemia, time in hyperglycaemia, and time in range for type 2 diabetes patients initially treated with multiple daily insulin injections (MDI) were investigated. Variables associated with hypoglycaemia in the current population were also identified.
\end{abstract}

Enhanced Digital Features To view enhanced digital features for this article go to https://doi.org/10.6084/ m9.figshare.9777764.

Electronic Supplementary Material The online version of this article (https://doi.org/10.1007/s13300019-00692-1) contains supplementary material, which is available to authorized users.

S. Sofizadeh $(\bowtie) \cdot$ A. F. Ólafsdóttir · S. Dahlqvist . M. Lind

Department of Medicine, NU-Hospital Group, Uddevalla, Sweden

e-mail: sheyda.sofizadeh@vgregion.se

S. Sofizadeh · A. F. Ólafsdóttir · S. Dahlqvist .

M. Lind

Department of Molecular and Clinical Medicine, University of Gothenburg, Gothenburg, Sweden

H. Imberg

Statistiska Konsultgruppen, Gothenburg, Sweden

H. Imberg

Department of Mathematical Sciences, Chalmers University of Technology and University of Gothenburg, Gothenburg, Sweden
Methods: Analyses were based on data from a previously performed double-blind, placebocontrolled trial in which 124 MDI-treated patients with type 2 diabetes were randomized to liraglutide or placebo. Masked continuous glucose monitoring (CGM) was performed at baseline and week 24 in 99 participants.

Results: The mean time in hypoglycaemia was similar for participants receiving liraglutide and those receiving placebo after 24 weeks of treatment. Mean time in target was greater in the liraglutide group than in the placebo group: 430 versus $244 \mathrm{~min} / 24 \mathrm{~h}(p<0.001)$ and 960 versus $695 \mathrm{~min} / 24 \mathrm{~h}(p<0.001)$ for the two glycaemic ranges considered, $4-7 \mathrm{mmol} / \mathrm{l}$ and $4-$ $10 \mathrm{mmol} / \mathrm{l}$, respectively. Mean time in hyperglycaemia was lower in the liraglutide group: 457 versus $723 \mathrm{~min} / 24 \mathrm{~h}(p=0.001)$ and 134

\section{Ekelund}

Novo Nordisk A/S, Vandtaarnsvej 114, 2860 Søborg, Denmark

\section{Ekelund · K. Filipsson · B. Ahrén}

Department of Clinical Sciences Lund, Lund

University, Lund, Sweden

I. Hirsch

School of Medicine, University of Washington,

Seattle, USA

\section{S. Sjöberg}

Department of Medicine, Karolinska Institute, Karolinska University Hospital Huddinge,

Stockholm, Sweden 
versus $264 \mathrm{~min} / 24 \mathrm{~h}(p=0.023)$ for the two cutoffs considered, $>10 \mathrm{mmol} / \mathrm{l}$ and $>14 \mathrm{mmol} / \mathrm{l}$, respectively. Lower mean glucose level, lower C-peptide, and higher glucose variability were associated with an increased risk of hypoglycaemia in both treatment groups. Higher proinsulin level was associated with a lower risk of hypoglycaemia in the liraglutide group.

Conclusion: For type 2 diabetes patients initially treated with MDI, introducing liraglutide had a beneficial effect on glucose profiles estimated by masked CGM. Mean glucose level, glycaemic variability, C-peptide, and proinsulin level influenced the risk of hypoglycaemia in this population.

Trial Registration: ClinicalTrials.gov, number (EudraCT nr: 2012-001941-42).

Funding: Novo Nordisk funded this study. The Diabetes Research Unit, NU-Hospital Group funded the journal's Rapid Service Fee.

Keywords: Continuous glucose monitoring; Hyperglycaemia; Hypoglycaemia; Liraglutide; Placebo; Randomized clinical trial; Time in range; Type 2 diabetes mellitus

\section{INTRODUCTION}

Optimal glycaemic control is required for patients with type 2 diabetes (T2D) to minimize the risk of secondary organ damage $[1,2]$. Although several glucose-lowering treatments are available, insulin therapy eventually becomes necessary for many patients with T2D. However, insulin treatment is associated with side effects including hypoglycaemia and weight gain $[1,3,4]$. Treatment options, for example the combination of insulin with

J. Tuomilehto

Department of Public Health Solutions, National

Institute for Health and Welfare, Helsinki, Finland

J. Tuomilehto

Department of Public Health, University of Helsinki, Helsinki, Finland

J. Tuomilehto

Diabetes Research Group, King Abdulaziz University, Jeddah, Saudi Arabia insulin-sparing agents to minimize both insulin dose and side effects, exist for patients with T2D and high insulin requirements [5]. The most advanced insulin regimen is generally multiple daily insulin injections (MDI) in persons with T2D, which is often used when other treatments are insufficient [6].

Liraglutide is a glucagon-like peptide-1 (GLP1) receptor agonist that is administered subcutaneously once daily [7]. GLP-1 exerts several physiological actions: it suppresses glucose-dependent glucagon, delays gastric emptying, and decreases appetite, and it has insulinotropic effects $[7,8]$. In earlier work, we found that, compared with placebo, adding liraglutide reduced $\mathrm{HbA1c}$ without increasing the risk of hypoglycaemia as estimated by capillary glucose levels in T2D patients treated with MDI [9]. However, HbA1c only provides information on mean glucose level, not the extent to which low and high glucose levels exist, nor glucose variability. Recently, there has been a greater focus on continuous glucose monitoring (CGM), which involves registering glucose levels every fifth minute, as it is believed that CGM is important if we are to fully understand the effects of glucose-lowering treatments on glucose levels [10]. Moreover, capillary glucose checks are not an optimal way of determining the risk of hypoglycaemia since they are highly dependent on self-monitoring by the patient when they feel symptoms of hypoglycaemia. Some patients may ingest carbohydrates without checking their glucose levels, they may not have their meter available, or they may not feel symptoms if their glucose levels are not very low. Common CGM metrics used to evaluate glucose-lowering treatments include time in target, time in range, time in hyperglycaemia, and time in hypoglycaemia.

Our aim in this study was to evaluate, using masked CGM, the effect of liraglutide on glucose levels as compared to placebo treatment in T2D patients treated with MDI. We also attempted to identify variables associated with time in hypoglycaemia in these patients. 


\section{METHODS}

\section{Patients and Study Procedures}

The study design and the main outcomes of the trial have previously been described in detail $[9,11,12]$. The study was approved by the ethics committee at the University of Gothenburg in accordance with the principles of the Declaration of Helsinki. All participants gave their written informed consent. Recruitment occurred between February 2013 and February 2014. The study was registered in the EudraCT database before it commenced (EudraCT No 2012-001941-42). The proposed design was a randomized, double-blind trial lasting 24 weeks that included patients with T2D and poor glycemic control despite being treated with MDI for at least six months.

Patients were randomized to liraglutide or placebo, which were titrated from $0.6 \mathrm{mg}$ during the first week to $1.2 \mathrm{mg}$ during the second week to $1.8 \mathrm{mg}$ from the third week onwards. When initiating and increasing the dose of liraglutide/placebo, the patients were advised to temporarily reduce the insulin dose if the blood glucose level was already on target in order to avoid the potential risk of hypoglycaemia. After the titration of liraglutide/placebo to $1.8 \mathrm{mg}$, the insulin dose was increased again to the original dose. Patients were advised to continue to adjust their insulin dose throughout the study as they had done previously.

The primary endpoint was the change in HbA1c from baseline to week 24. Secondary endpoints included the effects of treatment on body weight and total insulin dose.

In brief, inclusion criteria were T2D patients treated with MDI with or without metformin (patients with premixed insulin were excluded), $\mathrm{HbA} 1 \mathrm{c} \geq 58 \mathrm{mmol} / \mathrm{mol}$ and $\leq 102 \mathrm{mmol} / \mathrm{mol}$, BMI $27.5-45.0 \mathrm{~kg} / \mathrm{m}^{2}$. Overall, 124 participants were randomized $1: 1$ to subcutaneous liraglutide or placebo $[7,9]$.

Patients had subcutaneous sensors (Dexcom G4 PLATINUM system, San Diego, CA, USA) that measured glucose values every fifth minute. In the current study (MDI-Liraglutide 6), masked CGM data were analyzed to compare the effects of liraglutide and placebo on blood glucose levels. Thus, masked CGM was performed one week before randomization (run-in period), in week 12 , and before week 24 . When masked, the receiver did not display glucose values and instead stored them for downloading. In total, 57 patients in the liraglutide group and 50 patients in the placebo group had masked CGM data at week 12. Masked CGM data at week 24 were available for 52 patients in the liraglutide group and 47 patients in the placebo group. Liraglutide was added to insulin therapy according to an algorithm described previously $[9,11]$.

\section{Glycaemic measures}

We compared liraglutide treatment with placebo in terms of their effects on time in hypoglycaemia $(<3.9 \mathrm{mmol} / \mathrm{l}$ and $<3.0 \mathrm{mmol} / \mathrm{l})$, time in target (4-7 mmol/l), time in range (4$10 \mathrm{mmol} / \mathrm{l})$, and time in hyperglycaemia $(>10 \mathrm{mmol} / \mathrm{l}$ and $>14 \mathrm{mmol} / \mathrm{l})$. Estimations were performed for weeks 12 and 24; 24 weeks was considered the main follow-up period. For each glycaemic metric, estimations were performed per $24 \mathrm{~h}$, but they were also stratified into daytime (06:00-22:00 and 06:00-24:00) and nighttime (22:00-06:00 and 24:00-06:00) estimations. We also estimated the mean amplitude of glycaemic excursions (MAGE [13]) as a glucose variability measure.

\section{Variables Associated with Hypoglycaemia}

We estimated how time in hypoglycaemia at weeks 12 and 24 was related to mean glucose level as estimated by masked CGM during the corresponding time period. In addition, we evaluated possible baseline predictors for time in hypoglycaemia $(<3.0 \mathrm{mmol} / \mathrm{l}$ and $<3.9 \mathrm{mmol} / \mathrm{l}$, respectively) at week 24 . The following baseline variables were evaluated as potential predictors: age, sex, diabetes duration, HbA1c, mean glucose level and standard deviation (SD) measured by masked CGM, BMI, waist circumference, abdominal sagittal diameter, waist-hip ratio, fasting adiponectin level, fasting C-peptide level, fasting proinsulin level, metformin use, total 
daily insulin dose, percent meal insulin of total insulin dose, time in hypoglycaemia $(<3.0 \mathrm{mmol} / \mathrm{l})$, and diabetes treatment satisfaction status scale (DTSQ). For the predictors that were statistically significant in the liraglutide group at the 5\% level, we also evaluated whether their predictive abilities were significantly stronger in the liraglutide group compared with the placebo group through interaction analysis.

\section{Laboratory Analyses}

All laboratory analyses were performed at the Karolinska University Hospital, Stockholm, Sweden. HbA1c was measured according to the International Federation of Clinical Chemistry (IFCC) method, with all values converted according to the National Glycosylation Standard Program (NGSP) for dual reporting [14]. A commercially available ELISA kit from Mercodia $\mathrm{AB}$ was used to measure proinsulin.

\section{Statistics}

For descriptive purposes, data are presented here as mean (SD), median (minimum, maximum) for continuous variables and as number (\%) for categorical variables. For comparisons between groups, Fisher's nonparametric permutation test was used for continuous variables, Fisher's exact test for dichotomous variables, the Mantel-Haenszel chi-square test for ordered categorical variables, and the Pearson chi-square test for non-ordered categorical variables. For all tests, $p<0.05$ was considered statistically significant.

Analysis of the percent of time in hypoglycaemia in relation to the CGM mean and various baseline predictors was performed using a log-linear quasi-binomial model with robust standard errors, accounting for the positive, skewed, and heteroscedastic distribution of percent of time in hypoglycemia. In prediction analyses, the baseline variables were analyzed one at a time and further adjusted for time in hypoglycaemia at baseline and CGM mean. An interaction between the predictor and treatment group was included to test whether the effect of the predictor differed between treatment groups, in order to eliminate spurious correlations due to study-related causes and phenomena such as regression to the mean.

\section{RESULTS}

\section{Baseline Characteristics}

Baseline characteristics for the original cohort (FAS population), including all patients with at least one HbA1c measurement during follow-up $(n=122)$, are shown in Table 1 along with patients with CGM data at week $24(n=99)$. There were no clear differences between the FAS population and the currently evaluated population with CGM information in terms of age, sex, HbA1c, CGM glucose profiles, or other patient characteristics (Table 1).

For the cohort with CGM data, there were no significant differences between patients receiving liraglutide and those receiving placebo (Table 1); 19 (36.5\%) and 16 (34.0\%) were women, respectively. The mean age in both groups was 64 (8.2) years. Mean HbA1c was 73.6 (11.0) vs. $74.5(12.1) \mathrm{mmol} / \mathrm{mol}(8.9 \%(1.0) \mathrm{vs}$. 9.0\% (1.1\%), BMI was 33.8 (4.2) vs. 33.3 (3.9) $\mathrm{kg} / \mathrm{m}^{2}$, total insulin dose was $105.7 \mathrm{U}$ (47.2) vs. 107.2U (42.1), and number of insulin injections daily was $4.5(0.9)$ vs. $4.4(0.6)$ in the two groups, respectively.

\section{Overall Effects of Liraglutide and Placebo on Time in Hypoglycaemia, Time in Range, and Time in Hyperglycaemia}

At week 24 (end of the follow-up period), there was no difference in time in hypoglycaemia between patients receiving liraglutide and those receiving placebo (Table 2); mean time in hypoglycaemia $<3.9 \mathrm{mmol} / \mathrm{l}$ was 19.5 (34.2) vs. 19.3 (32.3) $\mathrm{min} / 24 \mathrm{~h}$, respectively, $p=0.97$, and mean time in hypoglycaemia $<3.0 \mathrm{mmol} / \mathrm{l}$ was 3.5 (7.5) vs. 4.3 (11.2) $\mathrm{min} / 24 \mathrm{~h}, p=0.67$.

Time in range $(4-10 \mathrm{mmol} / \mathrm{l})$ at week 24 was significantly greater for liraglutide-treated patients: 960 (346) vs. 695 (393) $\mathrm{min} / 24 \mathrm{~h}$ for liraglutide- and placebo-treated patients, 
Table 1 Demographics and baseline characteristics of 122 subjects in the ITT population and 99 subjects with masked CGM data obtained at 24 weeks of follow-up

\begin{tabular}{|c|c|c|c|c|c|c|}
\hline \multirow[t]{2}{*}{ Variable } & \multicolumn{3}{|c|}{ ITT population $(n=122)$} & \multicolumn{3}{|c|}{$\begin{array}{l}\text { Masked CGM data obtained at week } \\
24(n=99)\end{array}$} \\
\hline & $\begin{array}{l}\text { Liraglutide } \\
(n=63)\end{array}$ & $\begin{array}{l}\text { Placebo } \\
(n=59)\end{array}$ & $p$ value & $\begin{array}{l}\text { Liraglutide } \\
(n=52)\end{array}$ & $\begin{array}{l}\text { Placebo } \\
(n=47)\end{array}$ & $p$ value \\
\hline \multirow[t]{3}{*}{ Age (years) } & $63.8(8.2)$ & $63.6(7.7)$ & 0.88 & $64.4(8.2)$ & $64.1(7.1)$ & 0.89 \\
\hline & $\begin{array}{c}66.3(44.1 ; \\
78.0)\end{array}$ & $\begin{array}{l}65.0(38.9 \\
77.3)\end{array}$ & & $\begin{array}{c}67.2(44.1 \\
78.0)\end{array}$ & $\begin{array}{c}64.9 \\
77.3)\end{array}$ & \\
\hline & $n=63$ & $n=59$ & & $n=52$ & $n=47$ & \\
\hline Female & $23(36.5 \%)$ & $20(33.9 \%)$ & 0.91 & $19(36.5 \%)$ & $16(34.0 \%)$ & 0.96 \\
\hline White race & $62(98.4 \%)$ & $59(100.0 \%)$ & 0.33 & $52(100.0 \%)$ & $47(100.0 \%)$ & 1.00 \\
\hline Hispanic or Latino & $2(3.2 \%)$ & $0(0.0 \%)$ & 0.17 & $1(1.9 \%)$ & $0(0.0 \%)$ & 0.34 \\
\hline \multicolumn{7}{|l|}{ Smoking status } \\
\hline Never a smoker & $26(41.3 \%)$ & $21(35.6 \%)$ & & $20(38.5 \%)$ & $17(36.2 \%)$ & \\
\hline Former smoker & $29(46.0 \%)$ & $31(52.5 \%)$ & & $26(50.0 \%)$ & $25(53.2 \%)$ & \\
\hline Current smoker & $8(12.7 \%)$ & $7(11.9 \%)$ & 0.69 & $6(11.5 \%)$ & $5(10.6 \%)$ & 0.92 \\
\hline \multirow[t]{3}{*}{ Diabetes duration (years) } & $17.3(7.7)$ & $17.0(8.2)$ & 0.88 & $17.4(7.0)$ & $17.5(8.3)$ & 0.96 \\
\hline & $\begin{array}{c}16.0(4.0 \\
40.0)\end{array}$ & $\begin{array}{c}16.0(2.0 \\
35.0)\end{array}$ & & $\begin{array}{c}16.5(4.0 ; \\
36.0)\end{array}$ & $\begin{array}{c}17.0(2.0 \\
35.0)\end{array}$ & \\
\hline & $n=63$ & $n=59$ & & $n=52$ & $n=47$ & \\
\hline \multirow[t]{3}{*}{ Weight $(\mathrm{kg})$} & $98.8(14.1)$ & $99.8(14.8)$ & 0.70 & $99.7(13.5)$ & $99.9(14.8)$ & 0.95 \\
\hline & $\begin{array}{c}100.0(69.0 ; \\
134.9)\end{array}$ & $\begin{array}{c}96.0(72.5 \\
139.2)\end{array}$ & & $\begin{array}{c}100.8(71.9 ; \\
134.9)\end{array}$ & $\begin{array}{c}98.5(72.5 \\
139.2)\end{array}$ & \\
\hline & $n=63$ & $n=59$ & & $n=52$ & $n=47$ & \\
\hline \multirow[t]{3}{*}{ Height $(\mathrm{cm})$} & $171.3(10.4)$ & $172.7(10.0)$ & 0.47 & $172.0(10.4)$ & $173.0(9.8)$ & 0.62 \\
\hline & $\begin{array}{l}172.0(148.0 ; \\
192.0)\end{array}$ & $\begin{array}{l}173.0(145.0 ; \\
194.0)\end{array}$ & & $\begin{array}{l}174.0(148.0 \\
192.0)\end{array}$ & $\begin{array}{l}173.0(145.0 ; \\
191.0)\end{array}$ & \\
\hline & $n=63$ & $n=59$ & & $n=52$ & $n=47$ & \\
\hline \multirow[t]{3}{*}{ BMI $\left(\mathrm{kg} / \mathrm{m}^{2}\right)$} & $33.7(4.3)$ & $33.5(4.0)$ & 0.75 & $33.8(4.2)$ & $33.3(3.9)$ & 0.59 \\
\hline & $\begin{array}{l}33.3(27.3 \\
44.0)\end{array}$ & $\begin{array}{l}33.5(27.7 \\
43.0)\end{array}$ & & $\begin{array}{l}33.4(27.3 \\
44.0)\end{array}$ & $\begin{array}{l}33.5(27.7 \\
43.0)\end{array}$ & \\
\hline & $n=63$ & $n=59$ & & $n=52$ & $n=47$ & \\
\hline \multirow[t]{3}{*}{ Abdominal sagittal diameter $(\mathrm{cm})$} & $27.9(3.5)$ & $27.8(3.5)$ & 0.78 & $27.8(3.2)$ & $27.8(3.4)$ & 0.95 \\
\hline & $\begin{array}{c}27.5(20.5 ; \\
36.9)\end{array}$ & $\begin{array}{c}27.2(22.0 \\
36.7)\end{array}$ & & $\begin{array}{c}27.5(20.6 \\
35.0)\end{array}$ & $\begin{array}{c}27.3(22.0 \\
36.7)\end{array}$ & \\
\hline & $n=63$ & $n=58$ & & $n=52$ & $n=47$ & \\
\hline
\end{tabular}


Table 1 continued

\begin{tabular}{|c|c|c|c|c|c|c|}
\hline \multirow[t]{2}{*}{ Variable } & \multicolumn{3}{|c|}{ ITT population $(n=122)$} & \multicolumn{3}{|c|}{$\begin{array}{l}\text { Masked CGM data obtained at week } \\
24(n=99)\end{array}$} \\
\hline & $\begin{array}{l}\text { Liraglutide } \\
(n=63)\end{array}$ & $\begin{array}{l}\text { Placebo } \\
(n=59)\end{array}$ & $p$ value & $\begin{array}{l}\text { Liraglutide } \\
(n=52)\end{array}$ & $\begin{array}{l}\text { Placebo } \\
(n=47)\end{array}$ & $p$ value \\
\hline \multirow[t]{3}{*}{ Waist circumference $(\mathrm{cm})$} & $116.1(10.2)$ & $115.7(10.6)$ & 0.81 & $116.5(9.3)$ & $115.9(10.2)$ & 0.75 \\
\hline & $\begin{array}{l}116.0(95.0 \\
135.5)\end{array}$ & $\begin{array}{l}113.0(101.0 ; \\
144.8)\end{array}$ & & $\begin{array}{l}116.5(95.5 \\
135.5)\end{array}$ & $\begin{array}{l}113.0(101.0 \\
144.8)\end{array}$ & \\
\hline & $n=61$ & $n=57$ & & $n=50$ & $n=45$ & \\
\hline \multirow[t]{3}{*}{ Hip circumference $(\mathrm{cm})$} & $112.9(9.4)$ & $111.6(9.6)$ & 0.46 & $113.1(9.2)$ & $111.6(9.1)$ & 0.42 \\
\hline & $\begin{array}{l}111.0(97.0 \\
138.0)\end{array}$ & $\begin{array}{l}110.3(94.0 \\
139.0)\end{array}$ & & $\begin{array}{l}111.0(101.0 ; \\
138.0)\end{array}$ & $\begin{array}{l}110.3(94.0 \\
139.0)\end{array}$ & \\
\hline & $n=60$ & $n=58$ & & $n=50$ & $n=46$ & \\
\hline \multirow[t]{3}{*}{ Waist hip ratio } & $1.03(0.07)$ & $1.04(0.06)$ & 0.54 & $1.03(0.07)$ & $1.04(0.06)$ & 0.63 \\
\hline & $\begin{array}{l}1.04(0.82 \\
1.16)\end{array}$ & $\begin{array}{l}1.04(0.90 \\
1.22)\end{array}$ & & $\begin{array}{l}1.04(0.82 \\
1.16)\end{array}$ & $\begin{array}{l}1.04(0.90 \\
1.22)\end{array}$ & \\
\hline & $n=60$ & $n=57$ & & $n=50$ & $n=45$ & \\
\hline \multirow[t]{3}{*}{ Systolic blood pressure (mmHg) } & $137.9(16.8)$ & $133.7(13.7)$ & 0.14 & $138.6(16.3)$ & $134.3(13.1)$ & 0.16 \\
\hline & $\begin{array}{l}139.0(101.0 \\
180.0)\end{array}$ & $\begin{array}{l}134.0(104.0 \\
157.0)\end{array}$ & & $\begin{array}{l}139.5(101.0 ; \\
180.0)\end{array}$ & $\begin{array}{l}134.0(107.0 \\
155.0)\end{array}$ & \\
\hline & $n=63$ & $n=59$ & & $n=52$ & $n=47$ & \\
\hline \multirow[t]{3}{*}{ Diastolic blood pressure ( $\mathrm{mmHg}$ ) } & $73.5(12.7)$ & $74.9(8.5)$ & 0.48 & $72.8(12.7)$ & $74.1(8.5)$ & 0.55 \\
\hline & $\begin{array}{l}74.0(45.0 \\
103.0)\end{array}$ & $\begin{array}{l}76.0(54.0 \\
97.0)\end{array}$ & & $\begin{array}{l}73.0(45.0 \\
97.0)\end{array}$ & $\begin{array}{l}75.0(54.0 \\
90.0)\end{array}$ & \\
\hline & $n=63$ & $n=59$ & & $n=52$ & $n=47$ & \\
\hline \multirow[t]{3}{*}{ HbAlc (IFCC) $(\mathrm{mmol} / \mathrm{mol})$} & $74.6(10.8)$ & $74.4(12.0)$ & 0.92 & $73.6(11.0)$ & $74.5(12.1)$ & 0.71 \\
\hline & $\begin{array}{l}73.0(53.0 \\
103.0)\end{array}$ & $\begin{array}{l}73.0(54.0 \\
101.0)\end{array}$ & & $\begin{array}{l}72.5(53.0 \\
103.0)\end{array}$ & $\begin{array}{l}73.0(54.0 \\
101.0)\end{array}$ & \\
\hline & $n=63$ & $n=59$ & & $n=52$ & $n=47$ & \\
\hline \multirow[t]{3}{*}{ HbAlc (NGSP) (\%) } & $8.98(0.99)$ & $8.96(1.10)$ & 0.91 & $8.89(1.00)$ & $8.97(1.11)$ & 0.70 \\
\hline & $\begin{array}{l}8.83(7.00 \\
11.58)\end{array}$ & $\begin{array}{l}8.83(7.09 \\
11.39)\end{array}$ & & $\begin{array}{l}8.79(7.00 \\
11.58)\end{array}$ & $\begin{array}{l}8.83(7.09 \\
11.39)\end{array}$ & \\
\hline & $n=63$ & $n=59$ & & $n=52$ & $n=47$ & \\
\hline \multirow{3}{*}{$\begin{array}{l}\text { Fasting low-density lipoprotein (LDL) } \\
\text { cholesterol }(\mathrm{mmol} / \mathrm{l})\end{array}$} & $2.22(0.79)$ & $2.28(0.96)$ & 0.70 & $2.22(0.79)$ & $2.20(0.99)$ & 0.94 \\
\hline & $\begin{array}{l}2.10(0.20 \\
\quad 4.40)\end{array}$ & $\begin{array}{l}2.30(0.50 \\
4.80)\end{array}$ & & $\begin{array}{l}2.20(0.20 \\
4.40)\end{array}$ & $\begin{array}{l}2.15(0.50 \\
\quad 4.80)\end{array}$ & \\
\hline & $n=61$ & $n=53$ & & $n=51$ & $n=42$ & \\
\hline
\end{tabular}


Table 1 continued

\begin{tabular}{|c|c|c|c|c|c|c|}
\hline \multirow[t]{2}{*}{ Variable } & \multicolumn{3}{|c|}{ ITT population $(n=122)$} & \multicolumn{3}{|c|}{$\begin{array}{l}\text { Masked CGM data obtained at week } \\
24(n=99)\end{array}$} \\
\hline & $\begin{array}{l}\text { Liraglutide } \\
(n=63)\end{array}$ & $\begin{array}{l}\text { Placebo } \\
(n=59)\end{array}$ & $p$ value & $\begin{array}{l}\text { Liraglutide } \\
(n=52)\end{array}$ & $\begin{array}{l}\text { Placebo } \\
(n=47)\end{array}$ & $p$ value \\
\hline \multirow{3}{*}{$\begin{array}{l}\text { Fasting high-density lipoprotein (HDL) } \\
\text { cholesterol }(\mathrm{mmol} / \mathrm{l})\end{array}$} & $1.12(0.23)$ & $1.07(0.32)$ & 0.39 & $1.15(0.23)$ & $1.08(0.33)$ & 0.26 \\
\hline & $\begin{array}{l}1.10(0.70 \\
1.80)\end{array}$ & $\begin{array}{l}1.00(0.60 \\
2.80)\end{array}$ & & $\begin{array}{l}1.10(0.80 \\
1.80)\end{array}$ & $\begin{array}{l}1.05(0.70 \\
2.80)\end{array}$ & \\
\hline & $n=63$ & $n=58$ & & $n=52$ & $n=46$ & \\
\hline \multirow[t]{3}{*}{ Fasting triglycerides $(\mathrm{mmol} / \mathrm{l})$} & $1.87(1.11)$ & $2.15(1.59)$ & 0.27 & $1.74(1.01)$ & $2.12(1.62)$ & 0.17 \\
\hline & $\begin{array}{l}1.60(0.59 \\
6.50)\end{array}$ & $\begin{array}{l}1.65(0.56 \\
9.60)\end{array}$ & & $\begin{array}{l}1.45(0.59 \\
6.50)\end{array}$ & $\begin{array}{l}1.60(0.56 \\
9.60)\end{array}$ & \\
\hline & $n=63$ & $n=58$ & & $n=52$ & $n=46$ & \\
\hline \multirow[t]{3}{*}{ Fasting total cholesterol $(\mathrm{mmol} / \mathrm{l})$} & $4.18(0.92)$ & $4.24(1.00)$ & 0.73 & $4.17(0.97)$ & $4.19(1.02)$ & 0.90 \\
\hline & $\begin{array}{l}4.10(2.70 \\
7.90)\end{array}$ & $\begin{array}{l}4.10(2.40 \\
6.80)\end{array}$ & & $\begin{array}{l}4.10(2.70 \\
7.90)\end{array}$ & $\begin{array}{l}4.10(2.40 \\
6.60)\end{array}$ & \\
\hline & $n=63$ & $n=58$ & & $n=52$ & $n=46$ & \\
\hline \multirow[t]{3}{*}{ Fasting plasma glucose $(\mathrm{FPG})(\mathrm{mmol} / \mathrm{l})$} & $9.96(3.17)$ & $9.41(2.55)$ & 0.30 & $9.49(2.88)$ & $9.29(2.71)$ & 0.73 \\
\hline & $\begin{array}{l}9.40(4.20 \\
17.90)\end{array}$ & $\begin{array}{l}9.40(2.50 \\
19.60)\end{array}$ & & $\begin{array}{l}9.10(4.20 \\
17.90)\end{array}$ & $\begin{array}{l}9.20(2.50 \\
19.60)\end{array}$ & \\
\hline & $n=63$ & $n=59$ & & $n=52$ & $n=47$ & \\
\hline \multirow[t]{3}{*}{ CGM mean $(\mathrm{mmol} / \mathrm{l})$} & $10.9(2.3)$ & $10.7(2.2)$ & 0.56 & $10.7(2.3)$ & $10.7(2.2)$ & 0.97 \\
\hline & $\begin{array}{l}10.5(5.7 \\
16.6)\end{array}$ & $\begin{array}{l}10.0(6.9 ; \\
16.7)\end{array}$ & & $\begin{array}{c}10.3(5.7 ; \\
16.6)\end{array}$ & $\begin{array}{c}10.5(6.9 ; \\
15.1)\end{array}$ & \\
\hline & $n=62$ & $\mathrm{n}=57$ & & $\mathrm{n}=52$ & $\mathrm{n}=46$ & \\
\hline \multirow[t]{3}{*}{ CGM SD $(\mathrm{mmol} / \mathrm{l})$} & $2.98(0.71)$ & $2.97(0.79)$ & 0.94 & $2.96(0.72)$ & $2.97(0.80)$ & 0.95 \\
\hline & $\begin{array}{c}2.95(1.64 \\
4.84)\end{array}$ & $\begin{array}{c}2.77(1.72 \\
5.80)\end{array}$ & & $\begin{array}{c}2.95(1.64 \\
4.84)\end{array}$ & $\begin{array}{l}2.77(1.72 \\
5.80)\end{array}$ & \\
\hline & $n=62$ & $n=57$ & & $n=52$ & $n=46$ & \\
\hline \multirow[t]{3}{*}{ MAGE (mg/dl) } & $128.3(37.2)$ & $124.3(29.7)$ & 0.54 & $124.9(36.6)$ & $122.8(30.1)$ & 0.76 \\
\hline & $\begin{array}{l}124.4(63.6 \\
206.1)\end{array}$ & $\begin{array}{c}117.6(72.0 \\
197.0)\end{array}$ & & $\begin{array}{l}120.0(63.6 \\
206.1)\end{array}$ & $\begin{array}{c}116.0(72.0 \\
197.0)\end{array}$ & \\
\hline & $n=55$ & $n=53$ & & $n=48$ & $n=45$ & \\
\hline \multirow[t]{3}{*}{ Mean postprandial glucose level } & $12.0(3.0)$ & $11.2(3.0)$ & 0.20 & $11.7(3.0)$ & $11.5(3.0)$ & 0.70 \\
\hline & $\begin{array}{l}11.7(6.7 \\
20.6)\end{array}$ & $\begin{array}{c}11.2(5.9 ; \\
19.8)\end{array}$ & & $\begin{array}{c}11.6(6.7 ; \\
20.6)\end{array}$ & $\begin{array}{c}11.5(6.4 \\
19.8)\end{array}$ & \\
\hline & $n=59$ & $n=57$ & & $n=50$ & $n=45$ & \\
\hline
\end{tabular}


Table 1 continued

\begin{tabular}{|c|c|c|c|c|c|c|}
\hline \multirow[t]{2}{*}{ Variable } & \multicolumn{3}{|c|}{ ITT population $(n=122)$} & \multicolumn{3}{|c|}{$\begin{array}{l}\text { Masked CGM data obtained at week } \\
24(n=99)\end{array}$} \\
\hline & $\begin{array}{l}\text { Liraglutide } \\
(n=63)\end{array}$ & $\begin{array}{l}\text { Placebo } \\
(n=59)\end{array}$ & $p$ value & $\begin{array}{l}\text { Liraglutide } \\
(n=52)\end{array}$ & $\begin{array}{l}\text { Placebo } \\
(n=47)\end{array}$ & $p$ value \\
\hline \multirow[t]{3}{*}{ Treatment satisfaction status scale } & $26.5(7.9)$ & $27.8(6.9)$ & 0.34 & $26.7(7.7)$ & $27.6(7.1)$ & 0.58 \\
\hline & $\begin{array}{l}28.0(5.0 \\
36.0)\end{array}$ & $\begin{array}{l}29.0(4.0 \\
36.0)\end{array}$ & & $\begin{array}{l}28.0(5.0 \\
36.0)\end{array}$ & $\begin{array}{l}29.0(4.0 \\
36.0)\end{array}$ & \\
\hline & $n=62$ & $n=58$ & & $n=51$ & $n=47$ & \\
\hline Metformin users & $43(68.3 \%)$ & $43(72.9 \%)$ & 0.72 & $33(63.5 \%)$ & $34(72.3 \%)$ & 0.47 \\
\hline \multirow[t]{3}{*}{ Total daily basal insulin dose (units) } & $57.2(25.9)$ & $59.3(26.4)$ & 0.66 & $56.8(26.6)$ & $59.5(26.7)$ & 0.62 \\
\hline & $\begin{array}{c}54.0(12.0 \\
130.0)\end{array}$ & $\begin{array}{l}60.0(18.0 \\
130.0)\end{array}$ & & $\begin{array}{l}54.0(12.0 \\
130.0)\end{array}$ & $\begin{array}{l}60.0(18.0 \\
130.0)\end{array}$ & \\
\hline & $n=63$ & $n=59$ & & $n=52$ & $n=47$ & \\
\hline \multirow[t]{3}{*}{ Total daily meal insulin dose (units) } & $48.1(25.6)$ & $46.3(26.6)$ & 0.70 & $48.9(27.2)$ & $47.7(26.7)$ & 0.83 \\
\hline & $\begin{array}{l}40.0(12.0 \\
114.0)\end{array}$ & $\begin{array}{c}40.0(8.0 \\
165.0)\end{array}$ & & $\begin{array}{l}41.0(12.0 \\
114.0)\end{array}$ & $\begin{array}{l}42.0(11.0 \\
165.0)\end{array}$ & \\
\hline & $n=63$ & $n=59$ & & $n=52$ & $n=47$ & \\
\hline \multirow{3}{*}{$\begin{array}{l}\text { Total daily meal and basal insulin } \\
\text { (units) }\end{array}$} & $105.3(44.9)$ & $105.6(41.5)$ & 0.97 & $105.7(47.2)$ & $107.2(42.1)$ & 0.87 \\
\hline & $\begin{array}{l}100.0(28.0 \\
228.0)\end{array}$ & $\begin{array}{l}100.0(42.0 \\
230.0)\end{array}$ & & $\begin{array}{l}100.0(28.0 \\
228.0)\end{array}$ & $\begin{array}{l}104.0(42.0 \\
230.0)\end{array}$ & \\
\hline & $n=63$ & $n=59$ & & $n=52$ & $n=47$ & \\
\hline \multirow[t]{3}{*}{ Total number of insulin injections } & $4.46(0.88)$ & $4.42(0.62)$ & 0.89 & $4.46(0.94)$ & $4.40(0.61)$ & 0.83 \\
\hline & $\begin{array}{l}4.00(3.00 \\
9.00)\end{array}$ & $\begin{array}{l}4.00(3.00 \\
6.00)\end{array}$ & & $\begin{array}{l}4.00(3.00 \\
9.00)\end{array}$ & $\begin{array}{l}4.00(3.00 \\
6.00)\end{array}$ & \\
\hline & $n=63$ & $n=59$ & & $n=52$ & $n=47$ & \\
\hline \multicolumn{7}{|l|}{ Past diabetic complications } \\
\hline MI & $6(9.5 \%)$ & $10(16.9 \%)$ & 0.34 & $5(9.6 \%)$ & $9(19.1 \%)$ & 0.28 \\
\hline Stroke & $1(1.6 \%)$ & $0(0.0 \%)$ & 1.00 & $0(0.0 \%)$ & $0(0.0 \%)$ & 1.00 \\
\hline PCI & $5(7.9 \%)$ & $8(13.6 \%)$ & 0.48 & $4(7.7 \%)$ & 7 (14.9\%) & 0.41 \\
\hline Bypass surgery & $5(7.9 \%)$ & 7 (11.9\%) & 0.67 & $5(9.6 \%)$ & $4(8.5 \%)$ & 1.00 \\
\hline Retinopathy & $9(14.3 \%)$ & $14(23.7 \%)$ & 0.27 & $7(13.5 \%)$ & $12(25.5 \%)$ & 0.20 \\
\hline Amputation & $0(0.0 \%)$ & $1(1.7 \%)$ & 0.97 & $0(0.0 \%)$ & $1(2.1 \%)$ & 0.95 \\
\hline Past foot ulcer & $3(4.8 \%)$ & $4(6.8 \%)$ & 0.93 & $2(3.8 \%)$ & $3(6.4 \%)$ & 0.90 \\
\hline
\end{tabular}


Table 1 continued

\begin{tabular}{|c|c|c|c|c|c|c|}
\hline \multirow[t]{2}{*}{ Variable } & \multicolumn{3}{|c|}{ ITT population $(n=122)$} & \multicolumn{3}{|c|}{$\begin{array}{l}\text { Masked CGM data obtained at week } \\
24(n=99)\end{array}$} \\
\hline & $\begin{array}{l}\text { Liraglutide } \\
(n=63)\end{array}$ & $\begin{array}{l}\text { Placebo } \\
(n=59)\end{array}$ & $p$ value & $\begin{array}{l}\text { Liraglutide } \\
(n=52)\end{array}$ & $\begin{array}{l}\text { Placebo } \\
(n=47)\end{array}$ & $p$ value \\
\hline Current foot ulcer & $0(0.0 \%)$ & $0(0.0 \%)$ & 1.00 & $0(0.0 \%)$ & $0(0.0 \%)$ & 1.00 \\
\hline
\end{tabular}

$n(\%)$ is presented for categorical variables

Mean $(\mathrm{SD}) /$ median $(\min ; \max ) / n$ is presented for continuous variables

For comparisons between groups, Fisher's exact test was used for dichotomous variables, the Mantel-Haenszel chi-square test was used for ordered categorical variables, Pearson's chi-square test was used for non-ordered categorical variables, and Fisher's nonparametric permutation test was used for continuous variables

respectively, $p<0.001$ (Table 2 ). Regarding high glucose values ( $>10 \mathrm{mmol} / \mathrm{l})$, placebotreated patients spent $58 \%$ more time in this range: 723 (409) $\mathrm{min} / 24 \mathrm{~h}$ vs. 457 (362), $p=0.001$. Placebo-treated patients spent $97 \%$ more time with glucose levels higher than $14 \mathrm{mmol} / \mathrm{l}$.

Time in hypoglycaemia, time in range, and time in hyperglycaemia at week 12 are presented in Table 2. Similar patterns for the different glycaemic metrics were seen at weeks 12 and 24 for liraglutide-treated compared to placebo-treated patients except that liraglutidetreated patients spent more time in hypoglycaemia $<3.0 \mathrm{mmol} / \mathrm{l}$ at week $12(6.3$ [14.5] vs. 1.5 [4.5] $\mathrm{min} / 24 \mathrm{~h}, p=0.023)$.

\section{Times Spent in Diurnal and Nocturnal Hypoglycaemia}

Time in hypoglycaemia, time in target, time in range, and time in hyperglycaemia stratified into diurnal and nocturnal hours for patients receiving liraglutide and placebo at weeks 12 and 24 are shown in Table S1 of the Electronic supplementary material (ESM). There were similar patterns for diurnal and nocturnal hypoglycaemia to those seen for overall estimations per $24 \mathrm{~h}$ (Table 2 and Table S1 in the ESM). Time in range (4-10 $\mathrm{mmol} / \mathrm{l})$ was significantly greater for patients receiving liraglutide during both the daytime and the nighttime (Fig. 1), and time in hyperglycaemia ( $>10$ and $>14 \mathrm{mmol} / \mathrm{l}$ ) was significantly greater for placebo-treated patients. Regarding time in hypoglycaemia, there was a similar pattern to that seen in the overall analyses, with no differences in time spent in either diurnal or nocturnal hypoglycaemia $<3.9 \mathrm{mmol} / 1$ or $<3.0 \mathrm{mmol} / 1$ between the liraglutide-treated and placebotreated patients at week 24 . At week 12, time in nocturnal hypoglycaemia $<3.0 \mathrm{mmol} / \mathrm{l}$ was significantly greater in patients receiving liraglutide than in those receiving placebo, whereas there was no difference in time in diurnal hypoglycaemia (Table S1 in the ESM).

\section{Relationship of Mean Glucose Level with Hypoglycaemia}

There was a significant association of increased risk of time in hypoglycaemia with lower mean glucose level measured by masked CGM. This was evident in both the liraglutide and placebo groups for both the $<3.0 \mathrm{mmol} / \mathrm{l}$ and $<3.9 \mathrm{mmol} / \mathrm{l}$ glucose levels (Fig. 2).

At week 24 , there was a pattern of less hypoglycaemia $(3.9 \mathrm{mmol} / \mathrm{l})$ in the liraglutide group than in the placebo group at the same mean glucose level. At week 24, those on liraglutide who had relatively high mean glucose levels spent less time hypoglycaemia $<3.0 \mathrm{mmol} / 1$ (Fig. 2). At week 12, there was no difference in time in hypoglycaemia $<3.9 \mathrm{mmol} / \mathrm{l}$ between the treatment groups at a similar glucose level, whereas there was a tendency for more time in hypoglycaemia $<3.0 \mathrm{mmol} / \mathrm{l}$ in the liraglutide group (Fig. 2). 


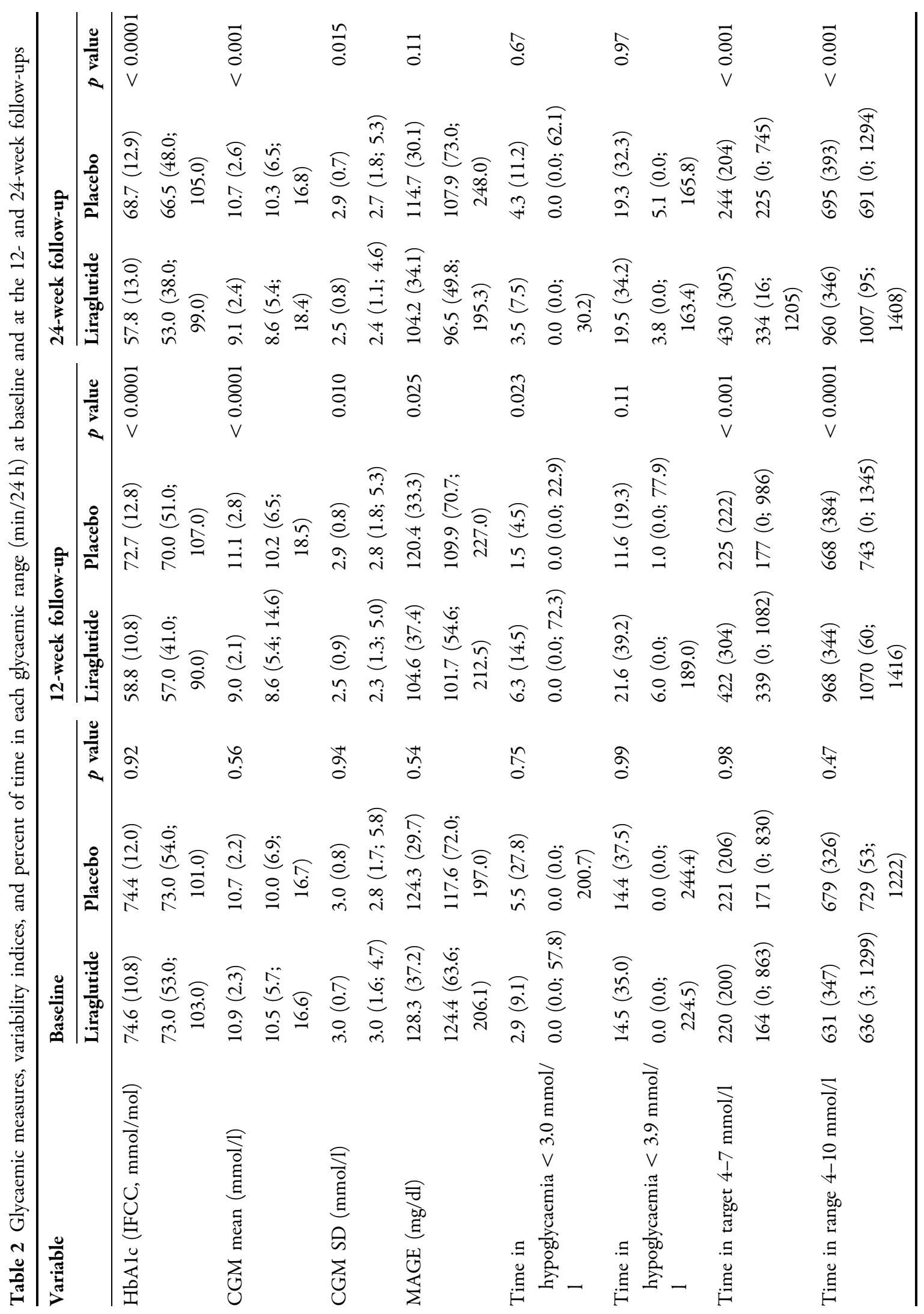



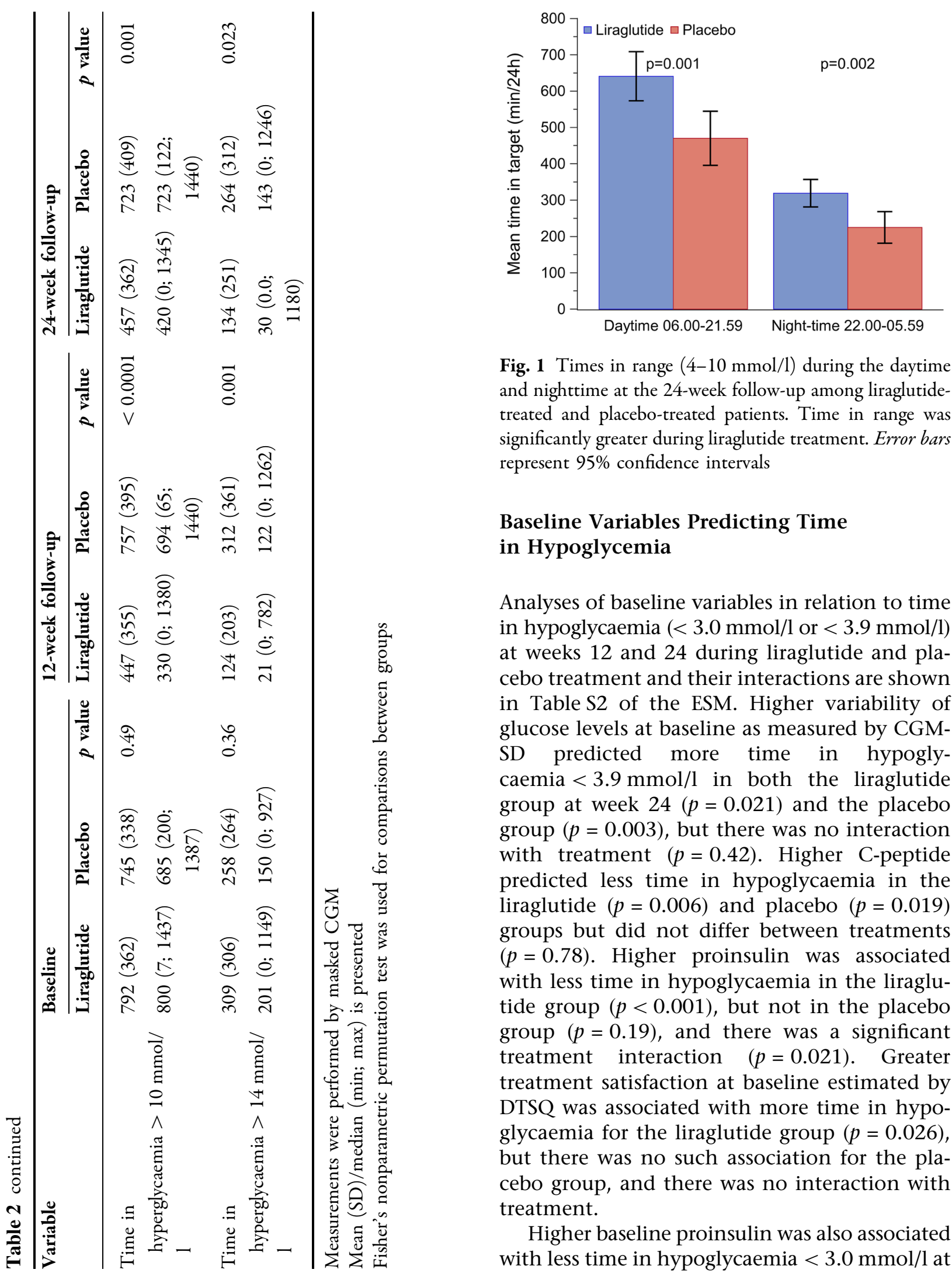

Fig. 1 Times in range $(4-10 \mathrm{mmol} / \mathrm{l})$ during the daytime and nighttime at the 24-week follow-up among liraglutidetreated and placebo-treated patients. Time in range was significantly greater during liraglutide treatment. Error bars represent $95 \%$ confidence intervals

\section{Baseline Variables Predicting Time in Hypoglycemia}

Analyses of baseline variables in relation to time in hypoglycaemia $(<3.0 \mathrm{mmol} / \mathrm{l}$ or $<3.9 \mathrm{mmol} / \mathrm{l})$ at weeks 12 and 24 during liraglutide and placebo treatment and their interactions are shown in Table 52 of the ESM. Higher variability of glucose levels at baseline as measured by CGMSD predicted more time in hypoglycaemia $<3.9 \mathrm{mmol} / \mathrm{l}$ in both the liraglutide group at week $24(p=0.021)$ and the placebo group ( $p=0.003)$, but there was no interaction with treatment $(p=0.42)$. Higher C-peptide predicted less time in hypoglycaemia in the liraglutide $(p=0.006)$ and placebo $(p=0.019)$ groups but did not differ between treatments $(p=0.78)$. Higher proinsulin was associated with less time in hypoglycaemia in the liraglutide group $(p<0.001)$, but not in the placebo group $(p=0.19)$, and there was a significant treatment interaction $(p=0.021)$. Greater treatment satisfaction at baseline estimated by DTSQ was associated with more time in hypoglycaemia for the liraglutide group $(p=0.026)$, but there was no such association for the placebo group, and there was no interaction with treatment.

Higher baseline proinsulin was also associated with less time in hypoglycaemia $<3.0 \mathrm{mmol} / \mathrm{l}$ at 

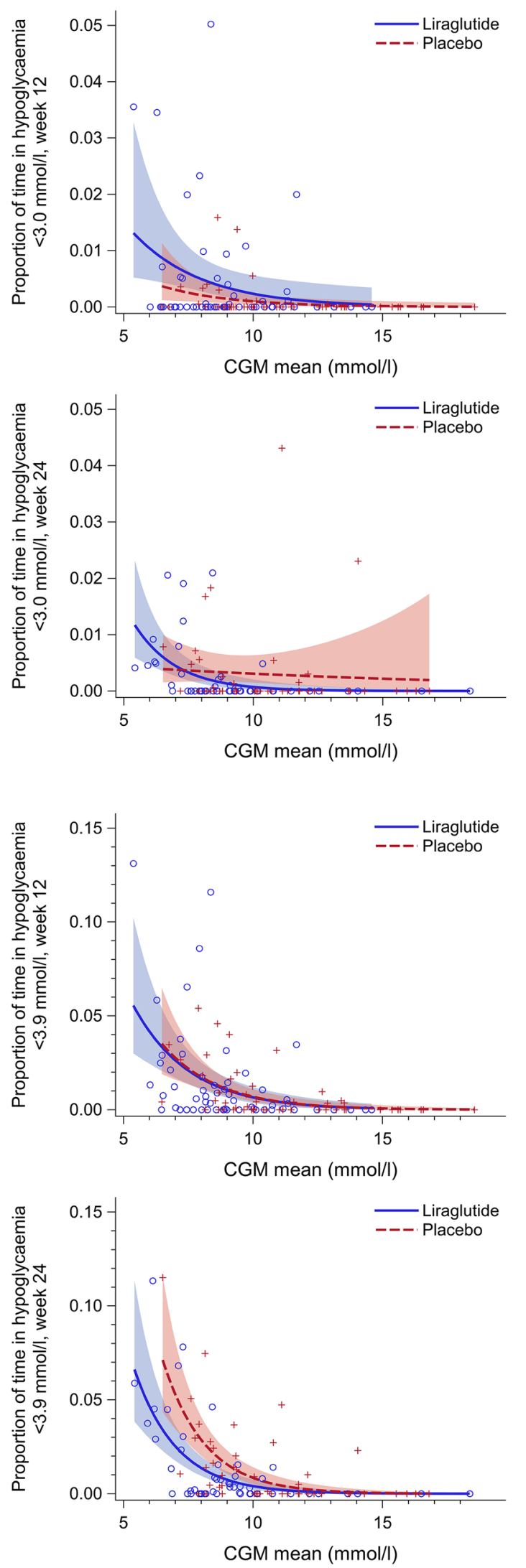

4Fig. 2 Percent time in hypoglycaemia $(<3.0$ or $<3.9$ $\mathrm{mmol} / \mathrm{l})$ vs CGM for the liraglutide-treated and placebotreated patients at the 12- and 24-week follow-ups. The risk of hypoglycaemia increased rapidly with decreasing glucose level in both treatment groups

week 24 in the liraglutide group ( $p=0.010)$, and there was a significant treatment interaction $(p=0.045)$. Higher mean baseline glucose measured via CGM was associated with less time in hypoglycaemia in the liraglutide group $(p=0.040)$, and there was a significant treatment interaction $(p=0.004)$. For the liraglutide group, baseline treatment satisfaction was associated with more time in hypoglycaemia $(p=0.011)$, and there was no treatment interaction $(p=0.77)$.

\section{DISCUSSION}

This randomized trial of liraglutide or placebo treatment in T2D patients already treated with MDI showed that glucose profiles estimated by masked CGM are significantly improved by liraglutide treatment. During liraglutide treatment, more time was spent at target glucose levels and less time at very high glucose levels. Time in hypoglycaemia was strongly associated with mean glucose level in both liraglutide- and placebo-treated patients. In addition, patients with lower fasting C-peptide levels and higher glycaemic variability had a greater risk of hypoglycaemia during MDI treatment with or without simultaneous liraglutide treatment. Those with higher proinsulin levels benefitted more from liraglutide treatment in terms of the reduction in time in hypoglycaemia.

Strengths of the current study include the randomized placebo-controlled design and repeated evaluations by masked CGM. Also, we evaluated variables associated with hypoglycaemia in both groups, as well as whether there was a treatment interaction. Limitations include the moderate size of the study, such that smaller effects may not have been detected. Another limitation is that not all patients had masked CGM data during follow-up (99 of 124 randomized patients). However, patient characteristics at baseline were similar for the 
liraglutide-treated and the placebo-treated patients with CGM data during follow-up.

It is of interest to understand whether liraglutide stabilizes glucose levels in T2D patients treated with MDI (i.e. whether it reduces the risk of hypoglycaemia). Some of our results support this hypothesis. At week 24 (the end of the study), patients treated with liraglutide had significantly more time in range without incurring more time in hypoglycaemia (below either 3.9 or $3.0 \mathrm{mmol} / \mathrm{l}$ ). We also attempted to investigate this issue by comparing the risk of hypoglycaemia at similar mean glucose levels in both treatment groups. This approach was limited, however, by the fact that few of the patients in the placebo group had low mean glucose levels, meaning that randomized comparisons were not possible. To definitively determine whether liraglutide reduces the risk of hypoglycaemia in MDI-treated T2D patients, a randomized trial obtaining similar mean glucose levels in both treatment arms is needed.

It has been thought that a major barrier to obtaining good glycaemic control during insulin treatment is that the risk of hypoglycaemia increases when the mean glucose level is reduced [15]. This view has, however, been challenged in certain studies both among T1D and T2D [16, 17]. Other factors, such as poor experiences with hypoglycaemia, adequate insulin dose adjustments, concomitant glucoselowering treatments, and unstable glucose metabolism may also be important when attempting to lower HbA1c and the corresponding risks of hypoglycaemia. However, the current study confirms that mean glucose level is strongly associated with risk of hypoglycaemia during MDI treatment in T2D patients with and without simultaneous incretin-based therapy. More time in hypoglycaemia has been associated with an increased risk of severe hypoglycaemia [18]. Hypoglycaemia has also been associated with an increased risk of cardiovascular disease and a lower quality of life [4, 19-21].

Longer duration of insulin treatment has been associated with risk of hypoglycaemia in T2D patients $[22,23]$. Longer diabetes duration has been associated with impaired glycaemic control and more extensive glucose-lowering treatments where MDI is generally a second- or third-line insulin regimen $[6,24]$. In the current study, mean diabetes duration was 17 years. On average, patients performed $>4$ insulin injections per day. Although all of the patients endogenously produced insulin (C-peptide $>0.1 \mathrm{nmol} / \mathrm{l}$ was an inclusion criterion), the level was essential for hypoglycaemia risk. The pancreas has likely a better possibility of adjusting insulin doses when exogenous insulin was not optimally administered. The lower risk of hypoglycaemia in liraglutide-treated patients with high proinsulin levels indicates that proinsulin level is associated with the strength of glucose-stabilizing effects. High proinsulin levels have also been associated with a greater HbA1c reduction by liraglutide [25]. Fasting proinsulin has been shown to be a marker of insulin resistance [26]. Whether proinsulin can be used as a marker for the glucose-stabilizing effects of liraglutide needs to be evaluated in further studies.

T2D patients treated with MDI likely benefit from a reduction in long-term complications due to improved 24-h glucose profiles. Improvements in both daytime and nighttime glucose profiles occurred in the current study. Time in range $(4-10 \mathrm{mmol} / \mathrm{l})$ was $38 \%$ higher with liraglutide treatment. Placebo-treated patients had $58 \%$ more time with glucose $>10 \mathrm{mmol} / \mathrm{l}$. Time with very high glucose levels $(>14 \mathrm{mmol} / \mathrm{l})$, which are of specific concern in relation to diabetes complications, was $97 \%$ greater with placebo treatment, corresponding to $>2 \mathrm{~h}$ more per 24 -h period. These estimates highlight the efficacy of liraglutide, which would not be so apparent if we only evaluated HbA1c. The glucose-lowering effects of liraglutide were obtained even though the patients receiving liraglutide treatment took considerably less insulin per $24 \mathrm{~h}$ [9].

The LEADER trial, which showed beneficial effects on cardiovascular disease of liraglutide treatment in T2D patients, did not include patients treated with MDI [27]. The current study shows that liraglutide treatment lowers glucose levels and reduces time in hypoglycaemia in T2D patients treated with MDI, and is therefore likely to reduce diabetes complications. 
Moreover, results from the current study imply that in clinical practice, healthcare professionals should pay attention to T2D patients receiving MDI treatment who have low mean glucose levels, low insulin production, and high glucose variability in relation to hypoglycaemia risk. Patients with higher proinsulin levels had a reduced risk of hypoglycaemia when they were treated with liraglutide, given the resulting mean glucose levels. Time may be essential for insulin titration in conjunction with treatment with GLP-1 analogues. In the current study, there were some indications that time in hypoglycaemia with liraglutide was greater at week 12 than at week 24 .

\section{CONCLUSION}

In conclusion, the current study using masked CGM data supports the notion that liraglutide is a valid treatment option for T2D patients treated with MDI. Introducing liraglutide in this patient population increased time in range and substantially reduced time with very high glucose levels without incurring any marked increase in the risk of hypoglycaemia. MDItreated T2D patients with low C-peptide levels and greater glycaemic variability were found to have a greater risk of hypoglycaemia. Proinsulin was related to the glucose-stabilizing effect of liraglutide.

\section{ACKNOWLEDGEMENTS}

We thank all the participants in the study. We thank all the participating sites, the local laboratories, the central laboratory at the Karolinska University Hospital, Gothia Forum for monitoring the trial, and Nordicinfu Care for training on masked continuous glucose monitoring and support with continuous glucose monitoring receivers and sensors.

Funding. Novo Nordisk provided financial support and study drugs for the study, but had no role in data collection, interpretation or the publication of the results. The journal's Rapid
Service Fee was covered by the Diabetes Research Unit, NU-Hospital Group.

Editorial Assistance. We thank Josh Murphy, an independent editing consultant, who performed language editing on an initial draft of the manuscript, funded by a grant from Novo Nordisk.

Authorship. All named authors meet the International Committee of Medical Journal Editors (ICMJE) criteria for authorship for this article, take responsibility for the integrity of the work as a whole, and have given their approval for this version to be published.

Authorship Contributions. ML, IH and JT designed the study. SD, KF and ML were involved in carrying out the study. HI analyzed the data and all authors took part in interpreting the data. SS and ML wrote the first draft of the manuscript. All authors revised the manuscript and approved the final version.

Disclosures. Sheyda Sofizadeh has been a consultant for Novo Nordisk and Pfizer. Sofia Dahlqvist's institution received grants from Novo Nordisk during the conduct of the study. Marcus Lind's institution received grants from Novo Nordisk during the conduct of the study. Marcus Lind has received honoraria or has been a consultant for AstraZeneca, DexCom, Eli Lilly, Novo Nordisk and Rubin Medical, participated in advisory boards for MSD and Novo Nordisk and received research grants from AstraZeneca and Dexcom outside the submitted work. Irl Hirsch has been a consultant for Abbott Diabetes Care, Adocia, Intarcia, Roche, Bigfoot and Valeritas. Karin Filipsson has been a speaker for NovoNordisk and Sanofi, and an advisory board member for MSD, NovoNordisk and Astra Zeneca. Jaako Tuomilehto has received grants from Bayer Pharma, Boehringer Ingelheim, Merck Serono and MSD outside the submitted work, has acted as a consultant, advisory board member or speaker for Merck Serono, Orion Pharma, Renova and MSD, and is a share owner in Orion Pharma. Magnus Ekelund is an employee of Novo Nordisk. Bo Ahrén has received lecture fees from Novartis, Merck, 
Sanofi and Novo Nordisk and has received grants from Novartis and Merck outside the submitted work. Stefan Sjöberg has occasionally been a consultant and received honoraria from Eli Lilly, Sanofi-Aventis, Novo Nordisk, Abbot Scandinavia, AstraZeneca, and Merck, Sharp \& Dohme, and has participated in advisory boards for Sanofi-Aventis, AstraZeneca, and Eli Lilly. Arndís F. Ólafsdóttir has nothing to disclose.

Compliance with Ethics Guidelines. The study was approved by the ethics committee at the University of Gothenburg in accordance with the principles of the Declaration of Helsinki. All participants gave written informed consent.

Data Availability. The datasets analyzed during the current study are available from the senior (last) author on reasonable request.

Open Access. This article is distributed under the terms of the Creative Commons Attribution-NonCommercial 4.0 International License (http://creativecommons.org/licenses/ by-nc/4.0/), which permits any noncommercial use, distribution, and reproduction in any medium, provided you give appropriate credit to the original author(s) and the source, provide a link to the Creative Commons license, and indicate if changes were made.

\section{REFERENCES}

1. UK Prospective Diabetes Study (UKPDS) Group. Intensive blood-glucose control with sulphonylureas or insulin compared with conventional treatment and risk of complications in patients with type 2 diabetes (UKPDS 33). Lancet. 1998;352:837-853.

2. Holman RR, Paul SK, Bethel MA, Matthews DR, Neil HA. 10-year follow-up of intensive glucose control in type 2 diabetes. $\mathrm{N}$ Engl $\mathrm{J}$ Med. 2008;359(15):1577-89.

3. Pi-Sunyer FX. The impact of weight gain on motivation, compliance, and metabolic control in patients with type 2 diabetes mellitus. Postgrad Med. 2009;121(5):94-107.
4. Gonder-Frederick LA, Clarke WL, Cox DJ. The emotional, social, and behavioral implications of insulin-induced hypoglycemia. Semin Clin Neuropsychiatry. 1997;2(1):57-65.

5. Lane W, Weinrib S, Rappaport J, Hale C. The effect of addition of liraglutide to high-dose intensive insulin therapy: a randomized prospective trial. Diabetes Obes Metab. 2014;16(9):827-32.

6. Davies MJ, D'Alessio DA, Fradkin J, et al. Management of hyperglycemia in type 2 diabetes, 2018. A consensus report by the American Diabetes Association (ADA) and the European Association for the Study of Diabetes (EASD). Diabetes Care. 2018;2018(41):2669-701.

7. Lind M. Incretin therapy and its effect on body weight in patients with diabetes. Prim Care Diabetes. 2012;6(3):187-91.

8. Drucker DJ, Nauck MA. The incretin system: glucagon-like peptide-1 receptor analogues and dipeptidyl peptidase- 4 inhibitors in type 2 diabetes. Lancet. 2006;368:1696-705.

9. Lind M, Hirsch IB, Tuomilehto J, et al. Liraglutide in people treated for type 2 diabetes with multiple daily insulin injections: randomised clinical trial (MDI Liraglutide trial). BMJ. 2015;351:h5364.

10. Danne T, Nimri R, Battelino $\mathrm{T}$, et al. International consensus on use of continuous glucose monitoring. Diabetes Care. 2017;40:1631-40.

11. Lind $\mathrm{M}$, Hirsch IB, Tuomilehto J, Dahlqvist $\mathrm{S}$, Torffvit O, Pehrsson NG. Design and methods of a randomised double-blind trial of adding liraglutide to control HbA1c in patients with type 2 diabetes with impaired glycaemic control treated with multiple daily insulin injections (MDI-Liraglutide trial). Prim Care Diabetes. 2015;9(1):15-22.

12. Ahmadi S, Filipsson K, Dimenäs H, et al. Effect of liraglutide on waist circumference, sagittal abdominal diameter and adiponectin levels in people with type 2 diabetes treated with multiple daily insulin injections. Evaluations from a randomized trial (MDI Liraglutide Study 5). Obes Sci Pract. 2019;5(2):130-40.

13. Baghurst PA. Calculating the mean amplitude of glycemic excursion from continuous glucose monitoring data. Diabetes Technol Ther. 2011;13(3):296-302.

14. Jeppsson JO, Kobold U, Barr J, et al. Approved IFCC reference method for the measurement of $\mathrm{HbA} 1 \mathrm{c}$ in human blood. Clin Chem Lab Med. 2002;40(1):78-89. 
15. DCCT Study Group. The effect of intensive treatment of diabetes on the development and progression of long-term complications in insulindependent diabetes mellitus. $\mathrm{N}$ Engl $\mathrm{J}$ Med. 1993;329:977-86.

16. Riddle MC. Effects of intensive glucose lowering in the management of patients with type 2 diabetes mellitus in the Action to Control Cardiovascular Risk in Diabetes (ACCORD) trial. Circulation. 2010;122(8):844-6.

17. Karges B, Rosenbauer J, Kapellen T, et al. Hemoglobin A1c levels and risk of severe hypoglycemia in children and young adults with type 1 diabetes from Germany and Austria: a trend analysis in a cohort of 37,539 patients between 1995 and 2012 . PLoS Med. 2014;11(10):e1001742.

18. Fiallo-Scharer R, Cheng J, Beck RW, et al. Factors predictive of severe hypoglycemia in type 1 diabetes: analysis from the Juvenile Diabetes Research Foundation continuous glucose monitoring randomized control trial dataset. Diabetes Care. 2011;34(3):586-90.

19. Stahn A, Pistrosch F, Ganz X, et al. Relationship between hypoglycemic episodes and ventricular arrhythmias in patients with type 2 diabetes and cardiovascular diseases: silent hypoglycemias and silent arrhythmias. Diabetes Care. 2014;37(2): 516-20.

20. Zhang Y, Wieffer H, Modha R, BalAR B, Pollack M, Krishnarajah G. The burden of hypoglycemia in type 2 diabetes: a systematic review of patient and economic perspectives. J Clin Outcomes Manag. 2010;17(12):547-57.
21. Frier BM, Schernthaner G, Heller SR. Hypoglycemia and cardiovascular risks. Diabetes Care. 2011;34(Suppl 2):S132-7.

22. Donnelly LA, Morris AD, Frier BM, et al. Frequency and predictors of hypoglycaemia in type 1 and insulin-treated type 2 diabetes: a population-based study. Diabetic Med. 2005;22(6):749-55.

23. Henderson JN, Allen KV, Deary IJ, Frier BM. Hypoglycaemia in insulin-treated type 2 diabetes: frequency, symptoms and impaired awareness. Diabetic Med. 2003;20(12):1016-21.

24. Lind M, Pivodic A, Cea-Soriano L, et al. Changes in $\mathrm{HbA} 1 \mathrm{c}$ and frequency of measuring HbA1c and adjusting glucose-lowering medications in the 10 years following diagnosis of type 2 diabetes: a population-based study in the UK. Diabetologia. 2014;57(8):1586-94

25. Dahlqvist S, Ahlén E, Filipsson K, et al. Variables associated with $\mathrm{HbA1c}$ and weight reductions when adding liraglutide to multiple daily insulin injections in persons with type 2 diabetes (MDI Liraglutide Trial 3). BMJ Open Diabetes Res Care. 2018;6(1):e000464.

26. Pfützner A, Kunt T, Hohberg C, et al. Fasting intact proinsulin is a highly specific predictor of insulin resistance in type 2 diabetes. Diabetes Care. 2004;27(3):682-7.

27. Marso SP, Daniels GH, Brown-Frandsen K, et al. Liraglutide and cardiovascular outcomes in type 2 diabetes. N Engl J Med. 2016;375(4):311-22. 